\title{
Contrast Enhancement for Improved Detection of Ananas Comosus from Aerial Imagery
}

\author{
Wan Nurazwin Syazwani R., Muhammad Asraf H., Megat Syahirul Amin Megat Ali
}

\begin{abstract}
This paper describes contrast enhancement method used in detecting Ananas Comosus from aerial images. The main challenge faced by many researchers is to deal with image under low contrast conditions. Under low contrast, target images of Ananas Comosus often blends with the background features such as leaves and ground, making detection difficult. This study is set out to investigate the usefulness of implementing contrast enhancement technique on existing image to improve detection performance. Aerial images under study were obtained at Simpang Renggam pineapple plantation using DJI Phantom 3 and subjected to two image pre-processing methods-intensity adjustment method and contrast-limited adaptive histogram equalization methods. As a control, these images were also evaluated against pre-processing method without any contrast enhancement. The performance of the techniques for detecting Ananas Comosus is then assessed using relative error, RE and mean absolute percentage, MAPE. The best results were obtained by using contrast-limited adaptive histogram equalization method; yielding RE of 0.125 and MAPE of $0.008 \%$ compared to intensity adjustment technique yielding maximum $R E$ of 0.286 and MAPE of $0.027 \%$. Meanwhile detection without image enhancement had resulted maximum RE of 0.250 and MAPE is $0.024 \%$.
\end{abstract}

Index Terms-Ananas Comosus, intensity adjustment, contrast-limited adaptive histogram equalization, detection, aerial image, enhancement, image processing

\section{INTRODUCTION}

$I$ MAGEenhancement is a pre-processing approach that improves the quality of digital images. It has been widely implemented for medical imaging, computer vision, digital photography, remote sensing imaging, and pattern recognition applications [1]. This process is important in image detection due to varying levels of light illumination, weather conditions, low quality image sensors, and non-uniform exposure [2][3] produces image of low contrast and contains noise with unnatural appearance. Generally, poor illumination affects

This manuscript is submitted on $23^{\text {th }}$ March 2020 and accepted on $25^{\text {th }}$ June 2020. This work was funded by the Faculty of Electrical Engineering, Universiti Teknologi MARA, and the Ministry of Education, Malaysia under the Fundamental Research Grant Scheme (600-IRMI/FRGS 5/3 (056/2019)).

W. N. S. Rahimi is with the Faculty of Electrical Engineering, Universiti Teknologi MARA, 40450 Shah Alam, Selangor, Malaysia (e-mail: azwinsyazwani13@gmail.com) M. A. Hairuddin is with the Faculty of Electrical Engineering, Universiti Teknologi MARA, Pasir Gudang Campus, 81750 Masai, Johor, Malaysia (e-mail: masraf@uitm.edu.my) M. S. A. Megat Ali is with the Microwave Research Institute, Universiti Teknologi MARA, 40450 Shah Alam, Selangor, Malaysia (e-mail: megatsyahirul@uitm.edu.my).

1985-5389/C 2021 The Authors. Published by UiTM Press. This is an open access article under the CC BY-NC-ND license (http://creativecommons.org/ licenses/by-nc-nd/4.0/). segmentation processes and detection function. For improved detection performance, suitable pre-processing method is required to enhance the quality of low contrast images. In the past, image enhancement has been implemented for human detection [4], face recognition [5], object detection [6] and medical imaging [7].

From existing agriculture-related studies, methods such as intensity adjustment technique [8], histogram equalization (HE) [9][10], adaptive histogram equalization (AHE) [11], and contrast-limited adaptive histogram equalization (CLAHE) [12] have been previously established. Studies have shown intensity adjustment to be relatively convenient, yet effective approach [13]. HE was shown to be most effective for grayscale images but is inefficient in coloured setting [13]. Meanwhile, AHE is used to enhance images in low light conditions with low contrast quality. Unfortunately, the method suffers from amplification of noise that exists in homogeneous regions. With this finding, it was concluded that a limiting approach is required for this contrast enhancement technique [14].

Meanwhile, CLAHE which is essentially an improvement of AHE [15] has shown to be robust to noise [14]. The approach involves focusing on localized regions rather than a complete image. Therefore, this paper proposes an improved image enhancement technique of contrast-limited adaptive histogram equalization (CLAHE), which solves some issues such as low contrast and noise. The main aim of this study is to investigate the differences in detection after pre-processing (1) without image contrast enhancement, (2) with intensity adjustment, and (3) with CLAHE techniques. In this study, the performance of intensity adjustment method and CLAHE method in detecting Ananas Comosus from aerial crop images was analysed. Both techniques are benchmarked against pre-processing methods without contrast enhancement.

\section{RESEARCH METHODS}

The study comprised of data collection, image extraction, contrast enhancement, background removal, morphological operation, bounding box implementation and object counting. All image processing and analysis by varying three contrast enhancement approaches as described below have been performed in the MATLAB environment. Fig. 1 shows the block diagram of the research methods by describing the mechanism used in this work. 


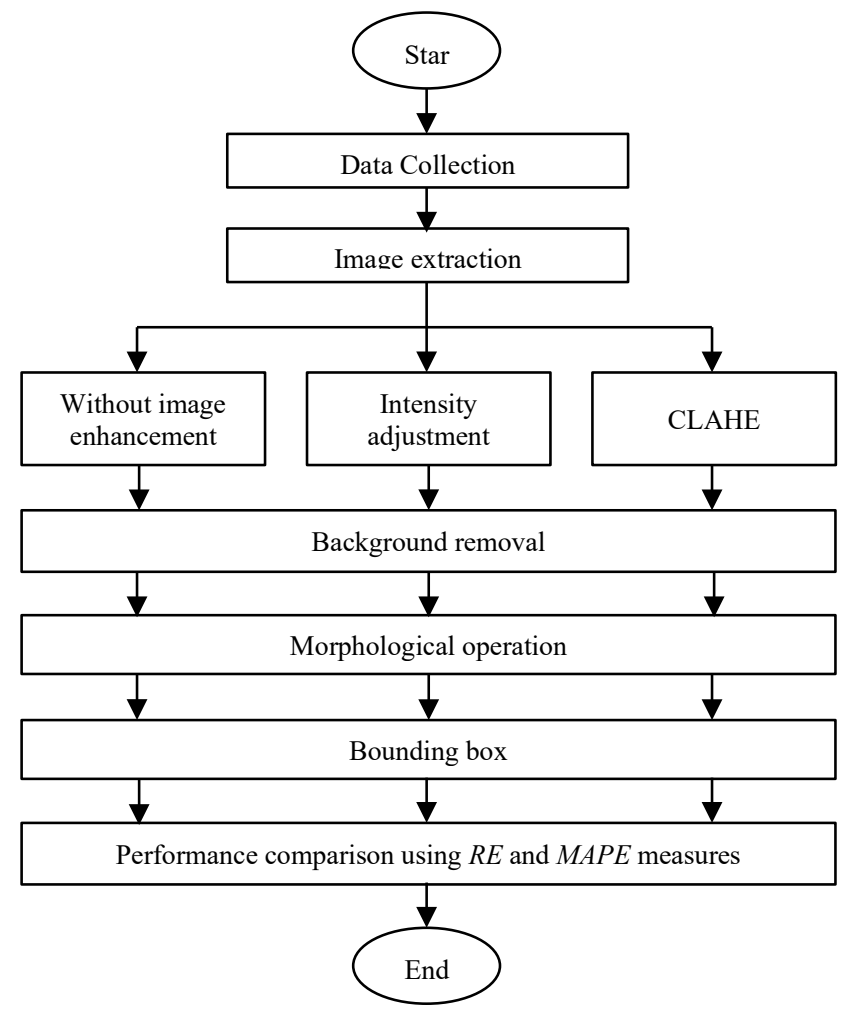

Fig. 1 The block diagram of research methods.

The data were first collected using the DJI Phantom 3 Advanced quadcopter with $4 \mathrm{~K}$ resolution RGB camera at pineapple plantation. The visual data werecaptured through aerial video recording of Simpang Renggam pineapple plantation in the state of Johor, Malaysia in March 2019. Then, the target images as depicted in Fig. 2 were extracted from video frame by frame using the loop code below.
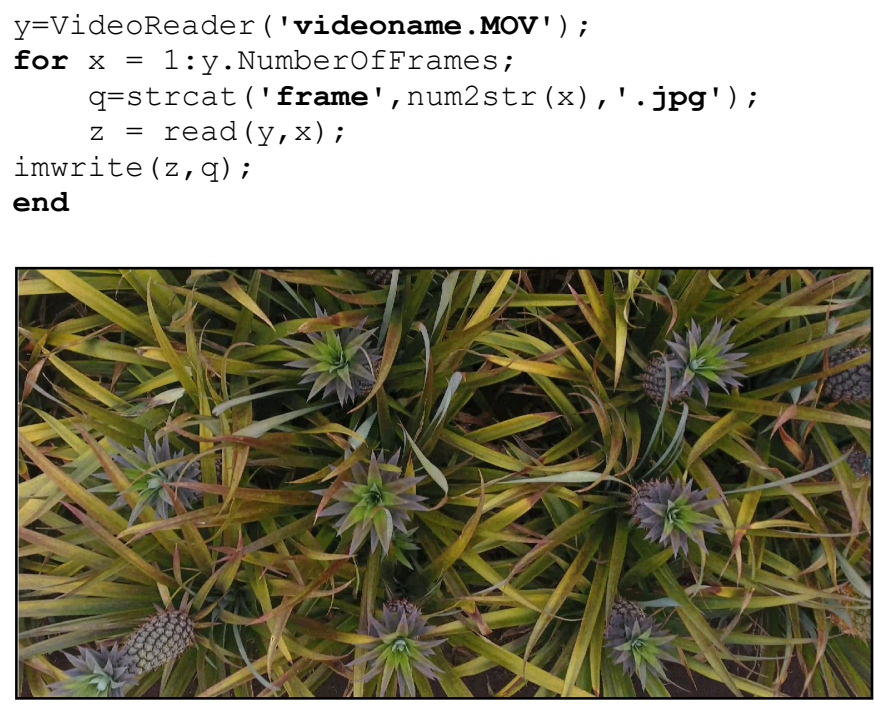

Fig. 2 Sample of extracted image from video recordings

The target image extraction works to retrieve the top view Ananas Comosus image frame by frame. From the video, the code converts a numeric array of frames to a string representation. The output format depends on the magnitudes of the original values. In order to generate frame by frame, the strings were concatenated horizontally. There are fifteen sample images selected randomly for further analysis. By applying image enhancement methods namely intensity adjustment method and CLAHE method, these techniqueswere compared to pre-processing without contrast enhancement. The rationale for selecting intensity adjustment as part of contrast enhancement analysis is that it provides a quick and simple method to improve the image contrast. After the contrast enhancement techniques have been applied, the pre-processed images were then filtered using HSV colour space to remove leaf and ground features from the Ananas Comosus images. Thresholding was performed in this space to create the most efficient mask for background removal.Subsequently,morphological operation is needed to remove some dotted in the binary images and to smooth the Ananas Comosus crown, thus filtering the existing noises. Dilation was also utilized to expand the white regions; hence effectively remove the black spots. In addition, the bounding box method was used in the final step to detect the crown of Ananas Comosus by accumulating the total number of boxes produced.

In order to evaluate the detection performance, a comparison was quantified between the pre-processed images without and with contrast enhancement using relative error, RE and mean absolute percentage error, MAPE. The assessments are based on minimum error of the undetected Ananas Comosus crown under variant illumination at the height of three metres. RE is defined as the ratio of absolute error between measured and actual detection and is expressed in equation (1) [15].

$R E=\frac{|M c-A c|}{M c} \times 100 \%$.

Mc denotes the number of successful detections by the bounding box technique, while $A c$ is the actual number of crowns in the image. Lower REvalue indicates higher detection accuracy. Meanwhile MAPE is a measure of prediction accuracy in statistical forecasting method [16]. It is an average of RE over the total number of samples, $\mathrm{N}$ and is expressed in equation (2).

$M A P E=\frac{1}{N} \sum_{i=1}^{N} R E_{i}$

Similar to RE, a lower MAPE would indicate higher level of detection accuracy. Lower value of RE and MAPE is indicative of better performance.

\section{RESUlTS AND DISCUSSION}

Fifteen samples are used to evaluate contrast enhancement methodstowards top view Ananas Comosus images. Observations are based on the contrast elements and formation of bounding boxes. Fig. 3-Fig. 5 show a sample of pre-processed image with and without contrast enhancement. 


\section{A. Pre-Processing without Contrast Enhancement}

Fig. 3 shows an unprocessed low contrast image. From the image, the crown of Ananas Comosus appears to be blending in with the background features of leaves and ground, making the detection process difficult. Additionally, the image histogram shows an uneven distribution at low brightness condition. The pixels values are highly clustered towards the left side, leaving few pixels for the top half of the intensity values. Contrast improvement can be achieved through histogram equalization, whereby having the pixels be distributed evenly over the whole intensity range. Past research has mentioned example of equalization method by performing image transformation in order to produce a flat histogram for the output image [14].
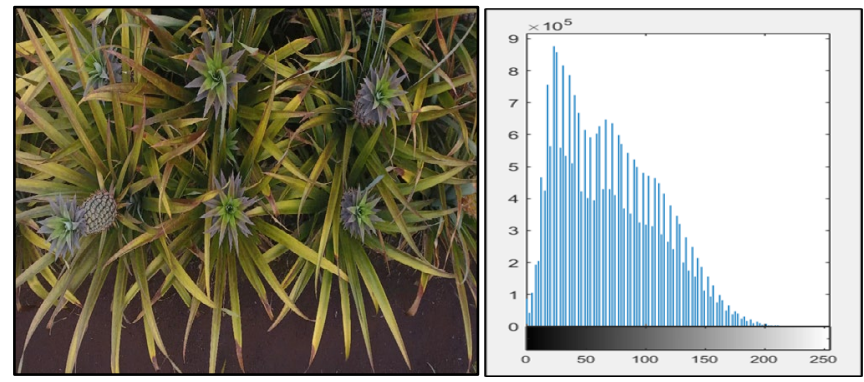

Fig. 3. (Left) Image without contrast enhancementand (Right) Histogram of original image.

\section{B. Pre-Processing with intensity adjustment}

For image enhancement, normally target for image improvement is specified such as higher signal-to-noise ratio or making a feature or colour to be perceived easily. The latter can typically be done by modifying the image colours, or intensities. Intensity adjustment is an image enhancement technique that maps an image's intensity values to a new range of values. With this transformation mapping, the entire intensity range of histogram can be filled, thus increasing the image contrast. For coloured image, the adjustment maps the value of intensity in an image within a range specified in RGB space [4]. The line of code below was used to adjust the image intensity values (also known as colormap). It maps the image intensity values, I to new values in J such that values between low in and high_in map to values between low_out and high_out. The values below low_in and above high_in are clipped; that is, values below low_in map to low_out, and those above high_in map to high_out. An empty matrix ([]) for [low_in high_in] or for [low_out high_out] to specify the default of [ 01$]$.

\footnotetext{
$\mathrm{J}=$ imadjust $\left(\mathrm{I},\left[\mathrm{l}_{\mathrm{OW}}\right.\right.$ in high_in], [low_out high_out], gamma)
}

The variable gamma in the code above specifies the shape of the curve describing the relationship between the values in I and J. If gamma is less than 1, the mapping is weighted toward higher (brighter) output values. If gamma is greater than 1, the mapping is weighted toward lower (darker) output values. If no value is specified, gamma defaults to 1 (linear mapping). The RGB values were manually adjusted to allow efficient background removal in HSV colour space. This results in clearer definition on the color of Ananas Comosuscrown, making detection easier. Fig. 4 shows the sample image and resulting histogram after being enhanced using intensity adjustment method.
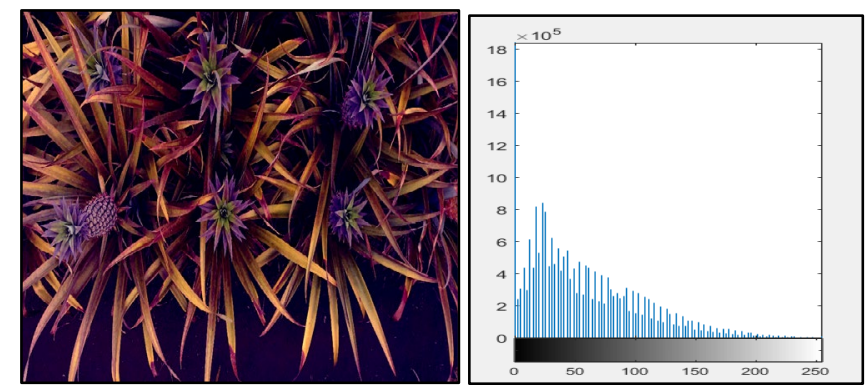

Fig.4..(Left)Contrast enhancement using intensity adjustment method and (Right)Histogram of intensity adjustment method.

\section{Pre-Processing with contrast-limited adaptive enhancement techniques}

CLAHE method is an improved version of AHE [1]. The method enhances the contrast of grayscale element of each tile so that the histogram of the output region matches the specified histogram. Fig. 5 shows the overall framework of CLAHE method. The image is initially divided into equally sized rectangular blocks, and histogram adjustment is performed in each block. Histogram adjustment process includes histogram creation, clipping, and redistribution. After performing equalization, CLAHE combines the neighboring blocks using bi-linear interpolation to eliminate artificially induced boundaries. CLAHE can limit the contrast by specifying a clip point to cut off the peak value in the histogram for each block. The clipped pixels are then distributed to each gray level. Higher clip point results in more contrast enhancement. equation (3) shows the calculation of clip point [2]:

$$
\beta=\frac{K}{L}\left(1+\frac{\alpha}{100} \operatorname{Smax}\right)
$$

where $K$ is the number of pixels in each block, $L$ is the dynamic range in this block, $S_{\max }$ is the maximum slope, and $\alpha$ is the clip factor. When $\alpha$ is set to 0 , the clip point would be $K / L$ so that the pixel in this block would be a constant. Substantial improvement in contrast occurs as $\alpha$ approaches 100 .

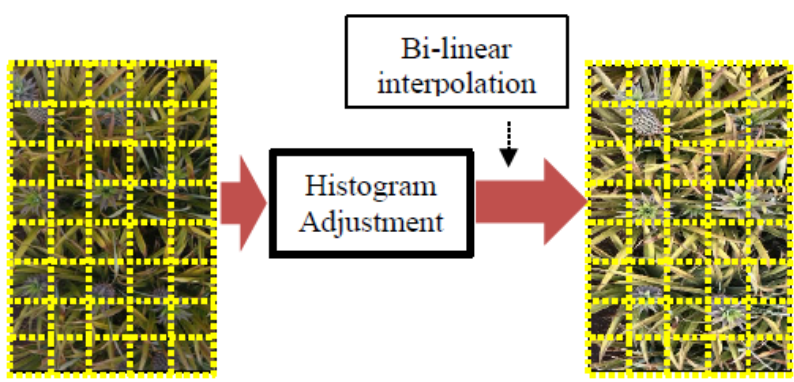

Fig. 5 Overall framework of CLAHE method.

For colour images, CLAHE method is performed by converting the indexed image into a true colour RGB format and then converting the RGB image further into $\mathrm{L}^{*} \mathrm{a}^{*} \mathrm{~b} *$ colour 
spaces. While maintaining the original colour values, the intensity of pixels is affected through manipulation of luminosity layer value, ' $L$ ', which will make the image appear lighter [4].Fig. 6 shows the sample image and resulting histogram after implementation of CLAHE method.
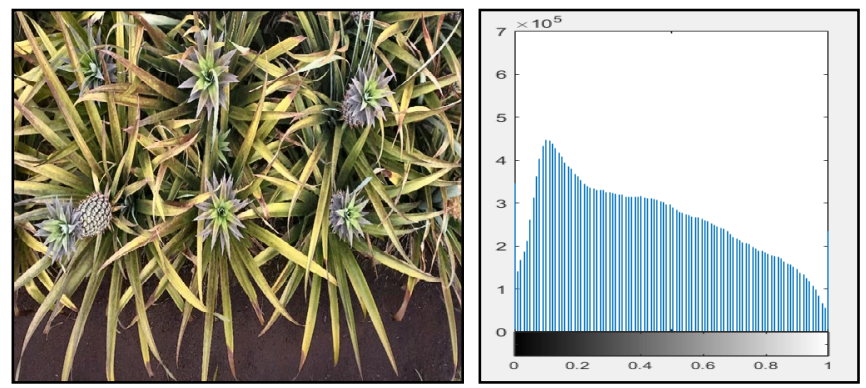

Fig. 6. (Left) Contrast enhancement using contrast-limited adaptive histogram equalization (Right) Histogram of contrast-limited adaptive histogram equalization technique.

Following contrast enhancement, images processed using intensity adjustment method and CLAHE method underwent similar background removal process using HSV colour space. These result in binary image which is further filtered from noise using morphological operation. The image is further processed into binary image to remove background features before crown detection. Dilation is utilized to expand the white regions; effectively removing black spots. Bounding box method is finally implemented to detect the crown of Ananas Comosus. Fig. 7 shows the sample of binary image after thresholding using HSV colour spaces. Fig. 8 and Fig. 9 respectively demonstrate the sample image following morphological operation and formation of bounding boxes [17] for detecting crown of Ananas Comosus.

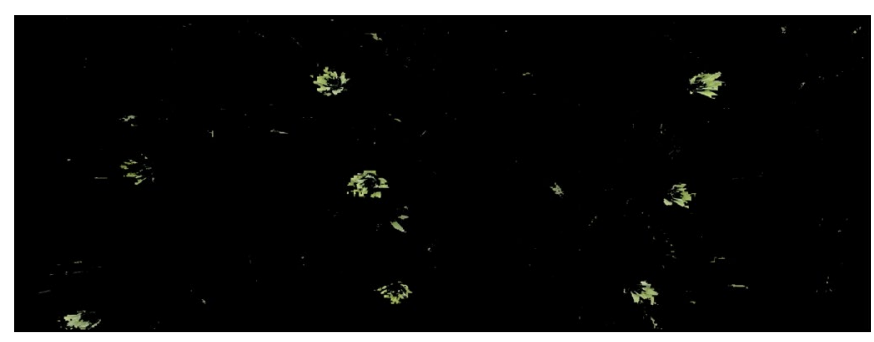

Fig. 7. Binary image after colour thresholding.

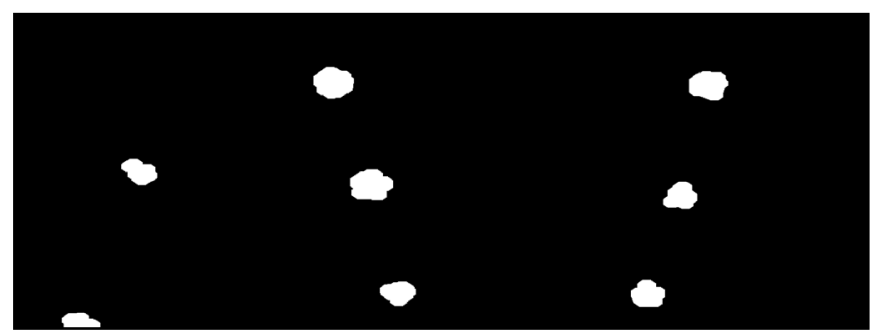

Fig. 8. Processed image after morphological operation.

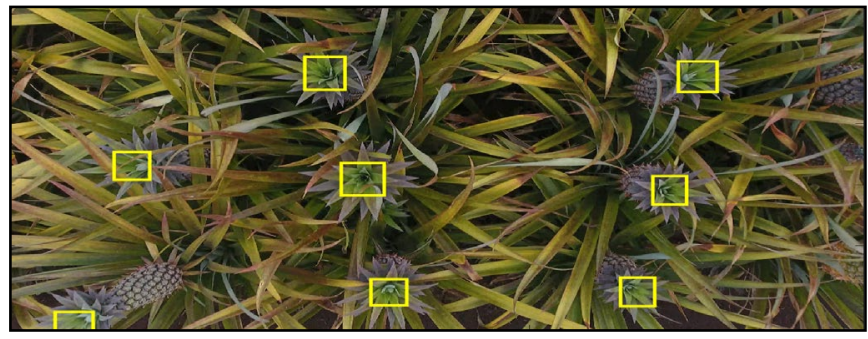

Fig. 9. Formation of bounding boxes.

\section{Performance measures}

The performance of the proposed contrast enhancement technique; intensity adjustment method and CLAHE method were compared against pre-processing without contrast enhancement. Table I compares the results in terms of RE and MAPE for fifteen sample images. In general, the increase in RE without contrast enhancement ranges from $0.00 \%$ to $0.25 \%$ withMAPE of $0.024 \%$. In the meantime, RE for pre processed images with intensity adjustment method ranges from $0.00 \%$ to $0.286 \%$ with MAPE of $0.027 \%$. The result obtained using this method shows a higher detection error than the pre-processed image without contrast enhancement; indicating that the method is relatively lower for the formation of bounding boxes around the crown of Ananas Comosus. On the other hand, the pre-processed images using CLAHE methodhave produced better results. RE ranges from $0.00 \%$ to $0.125 \%$ with MAPE at $0.008 \%$, the lowest of the three approaches. This indicate that CLAHE method exhibit superior contrast enhancement capabilities, allowing efficient formation of bounding boxes for crown detection even for low contrast images. Comparing the actual sample counts with the count output from each contrast enhancement method, consistent matches can be seen for most of the image samples $i$, except for $i$ of $7, \quad 10, \quad 12, \quad$ and 15 (refer to Fig. 10).

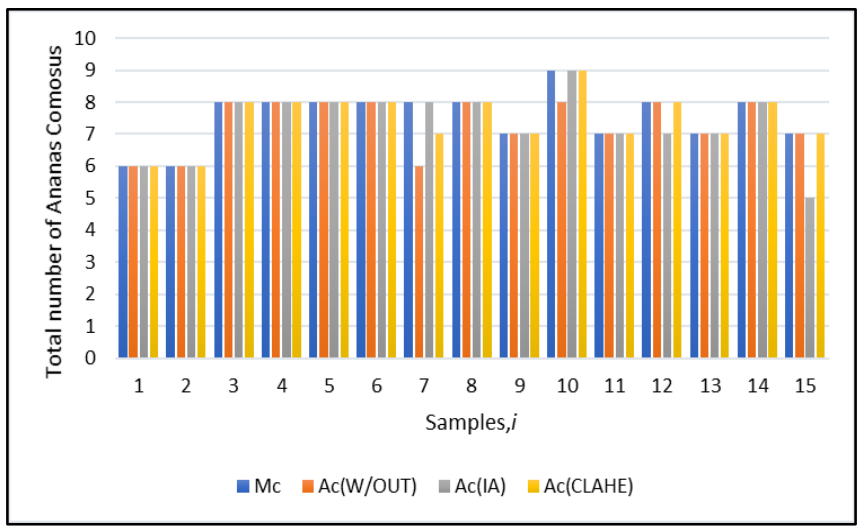

Fig. 10. Comparison of manual count and automatic count between detection produced without contrast enhancement (W/OUT), with intensity adjustment (IA), and contrast-limited adaptive histogram equalization (CLAHE).

Additionally, upon examining the RE values from each method (Fig. 11), CLAHE method is the lowest among the proposed approaches. Similar observation on MAPE values can be seen - CLAHE method results in lower MAPE 
TABLE I

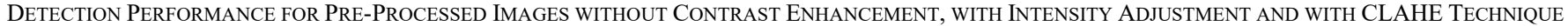

\begin{tabular}{|c|c|c|c|c|c|c|c|c|c|c|}
\hline \multirow{2}{*}{$i$} & \multirow{2}{*}{$M c$} & \multicolumn{3}{|c|}{ Without Contrast Enhancement } & \multicolumn{3}{|c|}{ Intensity Adjustment } & \multicolumn{3}{|c|}{ CLAHE } \\
\hline & & $A c$ & $R E$ & $M A P E$ & $A c$ & $R E$ & $M A P E$ & $A c$ & $R E$ & $M A P E$ \\
\hline 2 & 6 & 6 & 0 & \multirow{10}{*}{$0.024 \%$} & 6 & 0 & \multirow{10}{*}{$0.027 \%$} & 6 & 0 & \multirow{10}{*}{$0.008 \%$} \\
\hline 3 & 8 & 8 & 0 & & 8 & 0 & & 8 & 0 & \\
\hline 4 & 8 & 8 & 0 & & 8 & 0 & & 8 & 0 & \\
\hline 5 & 8 & 8 & 0 & & 8 & 0 & & 8 & 0 & \\
\hline 8 & 8 & 8 & 0 & & 8 & 0 & & 8 & 0 & \\
\hline 9 & 7 & 7 & 0 & & 7 & 0 & & 7 & 0 & \\
\hline 10 & 9 & 8 & 0.111 & & 9 & 0 & & 9 & 0 & \\
\hline 11 & 7 & 7 & 0 & & 7 & 0 & & 7 & 0 & \\
\hline 12 & 8 & 8 & 0 & & 7 & 0.125 & & 8 & 0 & \\
\hline 13 & 7 & 7 & 0 & & 7 & 0 & & 7 & 0 & \\
\hline
\end{tabular}

compared to other methods.Conclusively, CLAHE method offersthe best contrast enhancement for detection of Ananas Comosus. Future worksshould be devoted to development of more advance techniques to achieve error free detection. The challenges to overcome is that colour contrast between the target Ananas Comosus crown and the background is too similar.

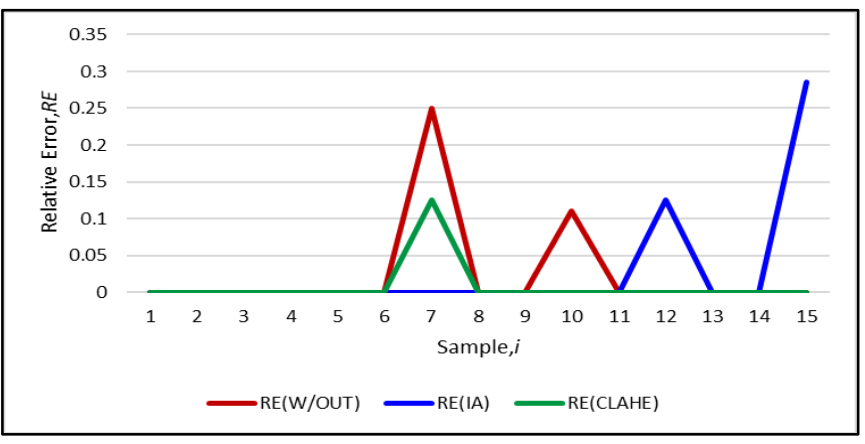

Fig. 11. Comparison of relative error between detection without contrast enhancement (W/OUT), intensity adjustment (IA), and contrast-limited adaptive histogram equalization (CLAHE).

Mean Absolute Percentage Error,MAPE

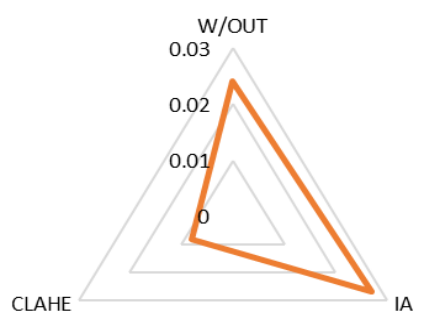

Fig. 12. Radar graph illustration to demonstrate the comparison of mean absolute percentage error between without contrast enhancement (W/OUT), intensity adjustment (IA), and contrast-limited adaptive histogram equalization (CLAHE).

Based on Fig. 12, the radar graph shows the comparison of MAPE between without contrast enhancement (W/OUT), intensity adjustment (IA), and contrast-limited adaptive histogram equalization (CLAHE). As conclude from this analysis, the MAPE value of the CLAHE method is lower than that of both techniques with $0.008 \%$, which is the best contrast enhancement technique for better detection of Ananas Comosus crowns.

\section{CONCLUSION}

This paper has presented two contrast enhancement method for improving detection of Ananas Comosus; intensity adjustment method and contrast-limited adaptive histogram equalization (CLAHE) method.The effectiveness of the proposed methods were also benchmarked against image preprocessing without contrast enhancement. From fifteen sample images that was acquired, crown detection performance for preprocessed images using intensity adjustment yielded the highest MAPE at $0.027 \%$, which also exceeded MAPE without contrast enhancement which is at $0.024 \%$. The best performance was obtained for pre-processed images with contrast-limited adaptive histogram equalization approach, yielding MAPE of $0.008 \%$.

\section{ACKNOWLEDGMENT}

This work is supported by the Faculty of Electrical Engineering, Universiti Teknologi MARA, and Ministry of Education, Malaysia under FRGS research grant, reference number of (600-IRMI/FRGS5/3(056/2019)). The members of the Precision Agri-food Technology Research Group, Faculty of Electrical Engineering, Universiti Teknologi MARA Johor branch are also thanked for supporting the research work.

\section{REFERENCES}

[1] C. Liu, X. Sui, Y. Liu, X. Kuang, G. Gu, and Q. Chen, "Adaptive contrast enhancement based on histogram modification framework," J. Mod. Opt., vol. 66, no. 15, pp. 1590-1601, Sep. 2019.

[2] Y. Chang, C. Jung, P. Ke, H. Song, and J. Hwang, "Automatic ContrastLimited Adaptive Histogram Equalization With Dual Gamma Correction,” IEEE Access, vol. 6, no. c, pp. 11782-11792, 2018.

[3] A. Singh and N. Kumar, "A Global-Local Contrast based Image 
Enhancement Technique based on Local Standard Deviation," Int. J. Comput. Appl., vol. 93, no. 2, pp. 8-12, May 2014.

[4] Neetu Mittal, "Automatic contrast enhancement of low contrast images using MATLAB," Int. J. Adv. Res. Comput. Sci., vol. 3, no. 1, pp. 333338,2012

[5] P. Musa, F. Al Rafi, and M. Lamsani, "A Review: Contrast-Limited Adaptive Histogram Equalization (CLAHE) methods to help the application of face recognition," in 2018 Third International Conference on Informatics and Computing (ICIC), 2018, pp. 1-6.

[6] A. S. Parihar and O. P. Verma, "Contrast enhancement using entropybased dynamic sub-histogram equalisation," IET Image Process., vol. 10, no. 11, pp. 799-808, Nov. 2016.

[7] M. Agarwal and R. Mahajan, "Medical Image Contrast Enhancement using Range Limited Weighted Histogram Equalization," Procedia Comput. Sci., vol. 125, no. 2017, pp. 149-156, 2018.

[8] I. Syarlina, B. Hj, and C. Ilias, "Intensity Adjustment Analysis of Underwater Images," J. Comput. Technol. Creat. Content, vol. 1, no. August, pp. 10-13, 2016.

[9] B. Oktavianto and T. W. Purboyo, "A Study of Histogram Equalization Techniques for Image Enhancement,” Int. J. Appl. Eng. Res., vol. 13, no. 2, pp. 1165-1170, 2018.

[10] R. B. Arif, M. M. R. Khan, and M. A. B. Siddique, "Digital Image Enhancement in Matlab: An Overview on Histogram Equalization and Specification," in 2018 International Conference on Innovation in Engineering and Technology (ICIET), 2018, no. November, pp. 1-6.

[11] Y. Zhu and C. Huang, "An Adaptive Histogram Equalization Algorithm on the Image Gray Level Mapping," Phys. Procedia, vol. 25, pp. 601608, 2012.

[12] R. P. Singh and M. Dixit, "Histogram Equalization: A Strong Technique for Image Enhancement," Int. J. Signal Process. Image Process. Pattern Recognit., vol. 8, no. 8, pp. 345-352, Aug. 2015.

[13] A. Namdeo and S. S. Bhadoriya, "A Review on Image Enhancement Techniques with its Advantages and Disadvantages," Int. J. Sci. Res. Technol., vol. 2, no. 5, pp. 171-182, 2016.

[14] D. Jyoti Bora, "Importance of Image Enhancement Techniques in Color Image Segmentation: A Comprehensive and Comparative Study," Indian J.Sci.Res, vol. 15, no. 1, pp. 115-131, 2017.

[15] Z. Lin, Z. Yingjie, D. Bochao, C. Bo, and L. Yangfan, "Welding defect detection based on local image enhancement," IET Image Process., vol. 13, no. 13, pp. 2647-2658, Nov. 2019.

[16] K. Djerriri, M. Ghabi, M. S. Karoui, and R. Adjoudj, "Palm Trees Counting in Remote Sensing Imagery Using Regression Convolutional Neural Network," in IGARSS 2018 - 2018 IEEE International Geoscience and Remote Sensing Symposium, 2018, vol. 2018-July, pp. 2627-2630.

[17] N. Meena, N. Aggarwal, S. Joshi, and S. Gupta, "Development of Detection, Counting and Yield Estimation Algorithm for Agricultural Products," Int. J. Eng. Res. Technol., vol. 3, no. 7, pp. 590-595, 2014.

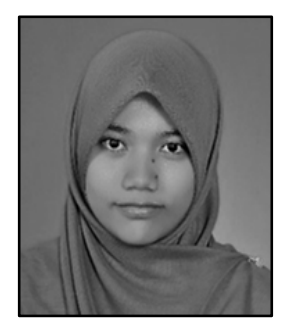

Wan Nurazwin Syazwani R. was born in Klang, Selangor in 1995. She received her Diploma in Electical Engineering (Instumentation) in 2015 and B.Eng (System) in 2018 from Universiti Teknologi MARA. She is currently a graduate research assistant at Universiti Teknologi MARA. Her interests include image processing and artificial intelligence with application to agriculture technology.

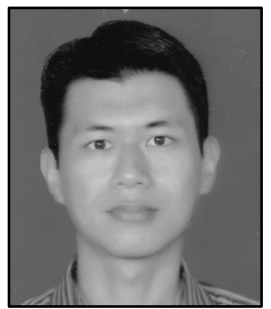

Muhammad Asraf H. received his $\mathrm{PhD}$ in Electrical Engineering from Universiti Teknologi MARA, Malaysia in 2014. He is currently a senior lecturer and research interests include image processing, artificial intelligenceand deep learning application.

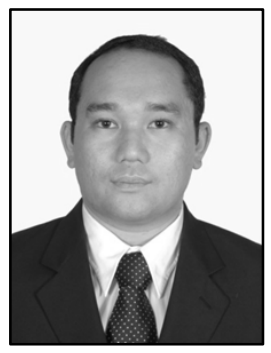

Megat Syahirul Amin Megat Ali received his B.Eng (Biomedical) from Universiti Malaya, M.Sc. in Biomedical Engineering from University of Surrey, and Ph.D. in Electrical Engineering from Universiti Teknologi MARA. $\mathrm{He}$ is currently a fellow at the Microwave Research Institute, Universiti Teknologi MARA. His research interests include digital signal processing and artificial intelligence, with applications to biomedical and radar sensing technology. 